\title{
Large Decreases in Standardized Uptake Values After Definitive Radiation Are Associated with Better Survival of Patients with Locally Advanced Non-Small Cell Lung Cancer
}

\author{
Jose L. Lopez Guerra ${ }^{1-3}$, Gregory Gladish ${ }^{4}$, Ritsuko Komaki ${ }^{1}$, Daniel Gomez ${ }^{1}$, Yan Zhuang ${ }^{5}$, and Zhongxing Liao ${ }^{1}$ \\ ${ }^{I}$ Department of Radiation Oncology, University of Texas M.D. Anderson Cancer Center, Houston, Texas; ${ }^{2}$ Department of Radiation \\ Oncology, Hospitales Universitarios Virgen del Rocio, Seville, Spain; ${ }^{3}$ Department of Medicine, Universitat Autònoma de Barcelona, \\ Barcelona, Spain; ${ }^{4}$ Department of Diagnostic Imaging, University of Texas M.D. Anderson Cancer Center, Houston, Texas; and \\ ${ }^{5}$ Department of Radiation Physics, University of Texas M.D. Anderson Cancer Center, Houston, Texas
}

We evaluated potential associations between maximum
standardized uptake value $\left(\mathrm{SUV}_{\max }\right)$ on ${ }^{18} \mathrm{~F}-\mathrm{FDG}$ PET before
and after radiation therapy $(\mathrm{RT})$ and survival outcomes for
patients with locally advanced non-small cell lung cancer.
Methods: Patients with stage III non-small cell lung cancer
$(n=49)$ who had undergone ${ }^{18} \mathrm{~F}-\mathrm{FDG}$ PET at the M.D.

Key Words: non-small cell lung cancer; radiation therapy; standardized uptake value; chemoradiation therapy; positron emission tomography

J Nucl Med 2012; 53:225-233

DOI: 10.2967/jnumed.111.096305 Anderson Cancer Center both before and up to 3.5 mo after undergoing radiochemotherapy were studied; exclusion criteria were patients with a history of thoracic surgery, $\mathrm{RT}$, or other cancer or those who had received a total radiation dose less than 60 Gy. We assessed associations between overall survival (OS) or disease-free survival (DFS) and postRT SUV $V_{\text {max }}$ and the extent of decrease in SUV $V_{\text {max }}$ in the primary tumor (PT) and regional lymph nodes (LNs). SUV max $_{\text {was }}$ assessed as a continuous variable by Cox proportional hazards regression analysis. Results: Univariate and multivariate analyses showed that having a high post-RT SUV $\max$ (either PT or LNs) was associated with a higher risk of death (univariate analyses: hazard ratio $[\mathrm{HR}]$ for PT SUV $\max , 1.27, P<0.0001$; HR for LN SUV max $1.32, P=0.004$ ) and disease recurrence (univariate analyses: HR for PT SUV $\max , 1.16, P=0.004$; HR for LN SUV $\max , 1.32, P=0.001)$. Moreover, after definitive RT, the greater the decrease in SUV $_{\text {max }}$ in the lesion that had the highest SUV $\mathrm{V}_{\max }$ at diagnosis, the longer the OS (HR, 0.06; $P=$ 0.002 ), DFS (HR, 0.03; $P=0.001$ ), local-regional control (HR, 0.04; $P=0.002)$, and distant metastasis-free survival (HR, 0.07; $P=0.028$ ). Conclusion: The post-RT SUV $\max$ in both the PT and the LNs was a predictor of survival-specifically, the higher the residual SUV $_{\max }$ after RT, the poorer the OS and DFS; and the greater the decrease in SUV max in the lesion with the highest $\mathrm{SUV}_{\max }$ at diagnosis, the longer the OS and DFS. This information should help to identify patients who are at high risk of recurrence and for whom additional treatments can be designed accordingly.

\footnotetext{
Received Jul. 29, 2011; revision accepted Sep. 26, 2011.

For correspondence or reprints contact: Zhongxing Liao, Department of Radiation Oncology, University of Texas M.D. Anderson Cancer Center, 1515 Holcombe Blvd., Unit 97, Houston, TX 77030.

E-mail: zliao@mdanderson.org

Published online Jan. 12, 2012.

COPYRIGHT ( 2012 by the Society of Nuclear Medicine, Inc.
}

$\mathbf{T}$ he current standard of care for patients with unresectable clinical stage III non-small cell lung carcinoma (NSCLC) is combined chemoradiation therapy. The median survival time after such treatment is approximately $17 \mathrm{mo}$ (1) — a significant improvement over historical treatment with radiation therapy (RT) or surgery alone but still quite poor. New treatment strategies for this disease are thus still urgently needed.

PET has had an increasing role in the initial staging and posttreatment evaluation of various types of cancer, including breast cancer, lymphoma, head and neck cancer, and NSCLC (2-8). The uptake of the glucose analog ${ }^{18} \mathrm{~F}-$ FDG by NSCLC tumor cells has been found to be useful for restaging at recurrence and for delineating radiotherapeutic targets (9-11). The standardized uptake value (SUV) in ${ }^{18} \mathrm{~F}-$ FDG PET before initial curative treatment has also been shown by some $(12,13)$, but not all $(14)$, to be a prognostic factor for survival outcomes. Therefore, the potential value of ${ }^{18} \mathrm{~F}-\mathrm{FDG}$ PET for predicting prognosis in advanced NSCLC before and after treatment still needs to be established.

A decline in ${ }^{18} \mathrm{~F}$-FDG uptake in the primary tumor or lymph nodes (LNs) after chemotherapy, RT, or both treatments for NSCLC is thought to reflect a reduction in the metabolic activity of the tumor and thus can be used to assess tumor response to therapy. The evaluation of the metabolic change after RT would be of considerable benefit given the difficulty of assessing whether the therapy worked with other imaging techniques (15). However, whether reductions in SUV in the primary tumor or LNs from before to after RT are linked with survival in patients with NSCLC 
remains uncertain. Also unclear is whether reductions in SUV could be associated with the likelihood of either local relapse or distant metastasis in such patients.

Hence, we undertook this study to assess potential associations between survival outcomes and the maximum SUV $\left(S_{\text {max }}\right)$ measured by ${ }^{18}$ F-FDG PET before and after therapy as well as the percentage of change in $\mathrm{SUV}_{\max }$ in the primary tumor, regional $\mathrm{LN}$ with the highest $\mathrm{SUV}_{\max }$, $\mathrm{SUV}_{\text {max }}$ sum of all regional LNs, target lesion (either primary tumor or LN) with the highest $\mathrm{SUV}_{\max }$, and $\mathrm{SUV}_{\max }$ sum of the primary tumor and all regional LNs in patients with inoperable stage III NSCLC who were candidates for curative therapy.

\section{MATERIALS AND METHODS}

\section{Patients}

This retrospective analysis was approved by the institutional review board of The University of Texas M.D. Anderson Cancer Center. Subjects were identified by a database search of patients with lung cancer treated with definitive radiochemotherapy in the Department of Radiation Oncology at M.D. Anderson Cancer Center between January 2007 and December 2008. Patients who were included in this study were those who had undergone an ${ }^{18} \mathrm{~F}$ FDG PET study done at M.D. Anderson both before and up to 3.5 mo after completing RT, who had no history of thoracic surgery or radiation therapy, who had no prior other cancer, and who had received a total radiation dose of 60 Gy or more. Ultimately, 49 patients met the selection criteria for this study. Disease had been staged according to the 2002 edition of the American Joint Committee on Cancer staging system (16). CT or ${ }^{18}$ F-FDG PET scans were obtained every 3 mo for the first 2 y after treatment, then every 6 mo for $5 \mathrm{y}$, and then annually thereafter to check for evidence of recurrence.

\section{F-FDG PET Technique}

Baseline (pretreatment) ${ }^{18} \mathrm{~F}-\mathrm{FDG}$ PET scans were obtained a median of $26 \mathrm{~d}$ before the start of RT. For patients who had received induction chemotherapy, we used the $S \mathrm{SV}_{\max }$ and clinical disease stages that had been assigned at initial diagnosis for our analysis. The median lag time between the end of the RT and the time of the ${ }^{18}$ F-FDG PET evaluation was 2.5 mo (range, 1-3.5 mo). For patients who had received adjuvant chemotherapy, the posttreatment scan was obtained after the chemotherapy. Scans were begun after a 6-h fast, with an intravenous injection of $370-555 \mathrm{MBq}$ of ${ }^{18} \mathrm{~F}-\mathrm{FDG}$, and emission and transmission scans were obtained $60 \mathrm{~min}$ afterward. All patients were required to have had a blood sugar level of $150 \mathrm{mg} / \mathrm{dL}$ or less before ${ }^{18} \mathrm{~F}$ FDG injection. Scans were obtained with a Discovery ST PET/CT scanner (GE Healthcare).

\section{SUV Measurements}

One experienced radiologist reviewed all ${ }^{18} \mathrm{~F}-\mathrm{FDG}$ PET images and obtained the $\mathrm{SUV}_{\max }$ for all patients. The $\mathrm{SUV}_{\max }$ in the gross primary tumor and in each LN was calculated and recorded from scans obtained before and after RT. Changes in SUV ( $\Delta$ SUV) after treatment were calculated with the following equation: $\Delta \mathrm{SUV}=$ ([SUVpre - SUVpost]/SUVpre) $\times 100$, where SUVpre and SUVpost denote pre- and posttreatment SUV, respectively. The primary tumor and LNs were manually drawn on the transaxial images around the focal ${ }^{18} \mathrm{~F}$-FDG uptake zone by referring to CT images before and after RT. For those cases considered to represent complete metabolic response (i.e., areas with ${ }^{18} \mathrm{~F}$-FDG uptake $\leq$ that in the aortic arch) or complete radiologic response (i.e., LN having a short axis of $<10 \mathrm{~mm}$ ), the $\mathrm{SUV}_{\text {max }}$ of the anatomic area corresponding to the pretreatment lesion was taken (Fig. 1). The SUVs of the primary tumor and LNs were calculated as SUV = activity concentration $(\mathrm{kBq}[\mu \mathrm{Ci}] / \mathrm{mL}) /($ injected dose $[\mathrm{MBq}$ $(\mathrm{mCi})] /$ body weight $[\mathrm{kg}]$ ). We used the maximum $\mathrm{SUV}_{\max }$ to avoid partial-volume effects (17). The $\mathrm{SUV}_{\max }$ is defined as the SUV of a 1-pixel region of interest corresponding to the maximum value in the primary tumor or $\mathrm{LN}$; thus, $\mathrm{SUV}_{\max }$ represents the value least affected by the partial-volume effect.

\section{Statistical Analysis}

Data were analyzed using Stata/SE 11.1 software (Stata Corp LP). Our goal was to investigate associations between the $\mathrm{SUV}_{\max }$ in the primary tumor or LNs and survival outcomes. For that purpose, we analyzed the $\mathrm{SUV}_{\max }$ before and after RT and the percentage of the change after RT in the following categories: the primary tumor, regional LN with the highest $\mathrm{SUV}_{\text {max }}, \mathrm{SUV}_{\max }$ sum of all regional LNs, target lesion with the highest $\mathrm{SUV}_{\max }$, and $\mathrm{SUV}_{\max }$ sum of primary tumor and all the regional LNs. Target lesions were defined as the primary tumor and any involved LNs. The Kaplan-Meier method was used to estimate the probability of overall survival (OS) and disease-free survival (DFS). Potential associations between the $\mathrm{SUV}_{\max }$ and survival outcomes were assessed in univariate analyses using the Cox proportional hazards model, and $\mathrm{SUV}_{\max }$ was evaluated as a continuous variable.

FIGURE 1. PET/CT scans of ${ }^{18} \mathrm{~F}-\mathrm{FDG}$ uptake by primary tumor and lymph nodes at diagnosis $(A)$ and after radiation therapy $(B)$. SUV $_{\text {aorta }}=$ SUV in aorta.

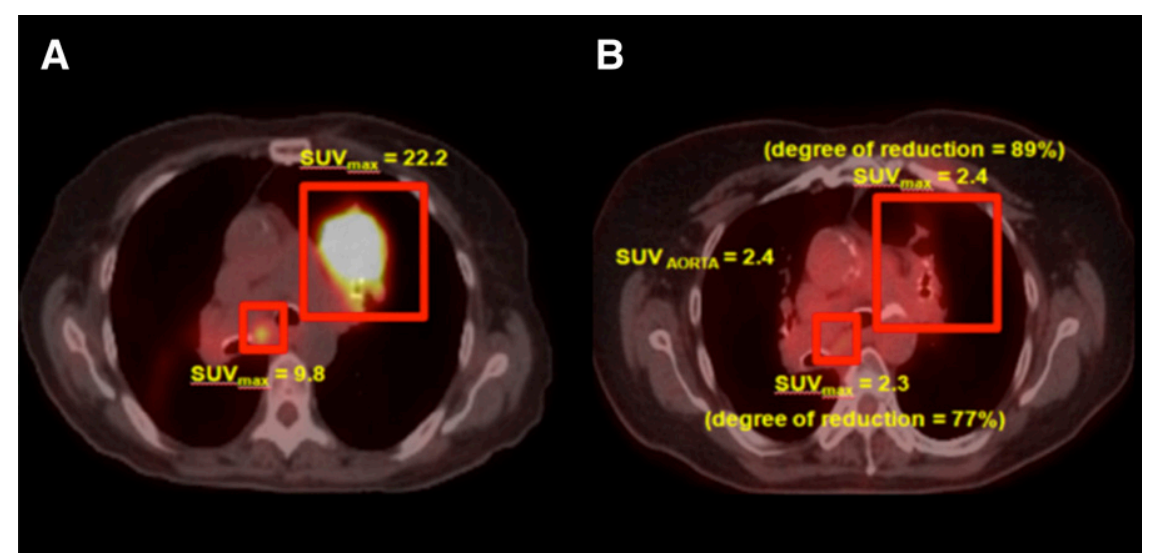


Because of the possible confounding effect of clinical factors on survival, associations found to be significant in the univariate analysis were adjusted by patient factors (age, sex, race, Karnofsky performance status, weight loss, and smoking habits), tumor factors (histology, stage), and treatment factors (type of chemotherapy, RT technique, and dose). Multivariate analyses were performed using a logistic regression model, with a stepwise backward elimination procedure. A $P$ value of 0.05 or less was considered statistically significant. Survival time was measured from the end of RT to the first occurrence of the considered event (death, local-regional recurrence [inside the radiation field, or both inside and outside the radiation field], or distant metastasis).

\section{RESULTS}

\section{Patients, Treatments, and Follow-up}

Patient characteristics are listed in Table 1. The median follow-up time for all patients was 25.5 mo (range, 3.9-49.4 mo). All patients had good performance status (Karnofsky performance score $\geq 70$ ) and underwent RT $5 \mathrm{~d}$ a week to a total dose of 63-74 Gy (median, 66 Gy) over 6-7 wk at 1.8-2.0 Gy per day. Forty-eight patients received platinum and taxane-based chemotherapy, 47 concurrent with RT and 1 sequentially. Of the 22 local-regional relapses, 16 occurred inside the radiation field; of the 18 distant metastases, 5 were in the brain.

\section{Survival Analyses}

$S U V_{\text {max }}$ for Primary Tumor. In 8 patients, distinguishing the ${ }^{18}$ F-FDG accumulation of the primary tumor from that of the LNs was difficult because the primary tumor extended to the hilar or subcarinal station. In those cases, the ${ }^{18}$ F-FDG accumulation was regarded as the SUV for the primary tumor. We observed that the higher the $\mathrm{SUV}_{\max }$ was after RT (Table 2), the shorter was the OS time $(P<$ 0.0001; Fig. 2) and DFS time ( $P=0.004$; Fig. 3). Figures 4 and 5 illustrate the effect of $\mathrm{SUV}_{\max }$ on OS and DFS, respectively, with the cut-off point of the $\mathrm{SUV}_{\max }$ after RT set at 3.7 (the 50th percentile for primary tumors); patients with an $S_{\text {max }}$ less than 3.7 had better OS and DFS than did those with an $\mathrm{SUV}_{\max }$ of 3.7 or greater (OS, $P=$ 0.0008; DFS, $P=0.0112$ ). We then investigated whether the interval between the end of RT and the PET scan had any influence on tumor response. For that purpose, we compared the $\mathrm{SUV}_{\text {max }}$ after RT in those patients who underwent PET 2.5 mo or less after RT (the median time between RT end date and PET scan date) and those who had the evaluation more than 2.5 mo after RT. We found no significant difference in the $\mathrm{SUV}_{\text {max }}$ after RT between both subgroups $(P=0.201)$. However, we did find that for the subgroup with the PET scan more than 2.5 mo after RT, the $\mathrm{SUV}_{\text {max }}$ after RT was associated with OS time $(P=0.021)$. To clarify the patterns of relapse, we evaluated localregional control (LRC) and distant metastasis-free survival (DMFS) rates in terms of $\mathrm{SUV}_{\max }$ and found that the higher the $\mathrm{SUV}_{\max }$ was after RT, the higher was the risk of localregional recurrence inside $(P=0.002)$ and both inside and outside $(P=0.002)$ the radiation field. However, no
TABLE 1

Patient Characteristics

\begin{tabular}{|c|c|}
\hline Characteristic & Value \\
\hline \multicolumn{2}{|l|}{ Age (y) } \\
\hline Median & 66 \\
\hline Range & $37-87$ \\
\hline \multicolumn{2}{|l|}{$\operatorname{Sex}(n)$} \\
\hline Male & $26(53.06)$ \\
\hline Female & $23(46.94)$ \\
\hline \multicolumn{2}{|l|}{ Race $(n)$} \\
\hline White & $45(91.84)$ \\
\hline Other* & $4(8.16)$ \\
\hline \multicolumn{2}{|l|}{ Karnofsky performance status ( $n$ ) } \\
\hline$<80$ & $8(16.33)$ \\
\hline 80 & $19(38.78)$ \\
\hline$>80$ & $22(44.90)$ \\
\hline \multicolumn{2}{|l|}{ Weight loss $(n)$} \\
\hline$<5 \%$ & $8(16.33)$ \\
\hline$\geq 5 \%$ & 41 (83.67) \\
\hline \multicolumn{2}{|l|}{ Smoking history $(n)$} \\
\hline Current smoker & $11(22.45)$ \\
\hline Quit smoking & 35 (71.43) \\
\hline Never smoked & $3(6.12)$ \\
\hline \multicolumn{2}{|l|}{ Histology $(n)$} \\
\hline Adenocarcinoma & 19 (38.78) \\
\hline Squamous & $19(38.78)$ \\
\hline NSCLC, not otherwise specified & $11(22.45)$ \\
\hline \multicolumn{2}{|l|}{ Clinical stage $(n)$} \\
\hline IIIA & $17(34.69)$ \\
\hline IIIB & $32(65.31)$ \\
\hline \multicolumn{2}{|l|}{ Induction chemotherapy ( $n$ ) } \\
\hline Yes & $10(20.41)$ \\
\hline No & 39 (79.59) \\
\hline \multicolumn{2}{|l|}{ Concurrent chemotherapy $(n)$} \\
\hline Yes & 47 (95.92) \\
\hline No & $2(4.08)$ \\
\hline \multicolumn{2}{|l|}{ Adjuvant chemotherapy $(n)$} \\
\hline Yes & 17 (34.69) \\
\hline No & $32(65.31)$ \\
\hline \multicolumn{2}{|l|}{ Radiation technique $(n)$} \\
\hline Intensity-modulated radiation therapy & $42(85.71)$ \\
\hline Proton-beam therapy & $7(14.29)$ \\
\hline \multicolumn{2}{|l|}{ Radiation dose $(n)$} \\
\hline Median & 66 \\
\hline Range & $63-74$ \\
\hline
\end{tabular}

*Other includes African Americans and Asians.

Data in parentheses are percentages.

significant associations with DMFS were found. All results that were statistically significant in the univariate analysis retained significance in the multivariate analysis (Table 3). Finally, for the primary tumor, the $\mathrm{SUV}_{\max }$ at diagnosis was not associated with any of the outcomes that were assessed.

$S U V_{\text {max }}$ for Regional LNs. Because some patients had more than one positive LN, we considered the single regional LN with the highest $\mathrm{SUV}_{\text {max }}$ and the $\mathrm{SUV}_{\max }$ sum of all PET-positive LNs separately for these analyses. No significant difference was found in the extent of SUV $\mathrm{max}_{\max }$ 
TABLE 2

Univariate Analyses of SUVs and Survival

\begin{tabular}{|c|c|c|c|c|c|c|c|c|c|c|c|c|}
\hline \multirow[b]{2}{*}{ Variable } & \multirow[b]{2}{*}{ Median } & \multirow[b]{2}{*}{$\begin{array}{l}\text { Interquartile } \\
\text { range }\end{array}$} & \multicolumn{2}{|c|}{ OS } & \multicolumn{2}{|c|}{ DFS } & \multicolumn{2}{|c|}{ LRC inside field } & \multicolumn{2}{|c|}{$\begin{array}{l}\text { LRC inside }+ \\
\text { outside field }\end{array}$} & \multicolumn{2}{|c|}{$\begin{array}{l}\text { Distant } \\
\text { metastasis- } \\
\text { free survival }\end{array}$} \\
\hline & & & $\begin{array}{l}\text { Hazard } \\
\text { ratio }\end{array}$ & $P$ & $\begin{array}{l}\text { Hazard } \\
\text { ratio }\end{array}$ & $P$ & $\begin{array}{l}\text { Hazard } \\
\text { ratio }\end{array}$ & $P$ & $\begin{array}{l}\text { Hazard } \\
\text { ratio }\end{array}$ & $P$ & $\begin{array}{l}\text { Hazard } \\
\text { ratio }\end{array}$ & $P$ \\
\hline \multicolumn{13}{|l|}{ Primary tumor } \\
\hline SUV $_{\max }$ at diagnosis & 14 & $10.6-16.9$ & 1.03 & 0.368 & 0.99 & 0.769 & 1.01 & 0.837 & 1.01 & 0.838 & 0.93 & 0.191 \\
\hline $\mathrm{SUV}_{\max }$ after RT & 3.7 & $2.7-5.5$ & 1.27 & $<0.0001$ & 1.16 & 0.004 & 1.19 & 0.002 & 1.17 & 0.002 & 1.16 & 0.066 \\
\hline Reduction (\%) in $\mathrm{SUV}_{\max }$ & 70 & $44-80$ & 0.53 & 0.158 & 0.62 & 0.229 & 0.52 & 0.159 & 0.60 & 0.257 & 0.52 & 0.242 \\
\hline \multicolumn{13}{|l|}{ Regional LN* } \\
\hline SUV $_{\max }$ at diagnosis & 11 & $6.4-15.4$ & 0.99 & 0.826 & 1.01 & 0.842 & 1.02 & 0.582 & 1.01 & 0.692 & 0.94 & 0.266 \\
\hline SUV $_{\max }$ after RT & 3.1 & $2.4-4.1$ & 1.32 & 0.004 & 1.32 & 0.001 & 1.26 & 0.011 & 1.29 & 0.002 & 1.11 & 0.533 \\
\hline Reduction in $\mathrm{SUV}_{\max }$ & 64 & $32-80$ & 0.19 & 0.070 & 0.48 & 0.330 & 0.65 & 0.681 & 0.55 & 0.481 & 0.26 & 0.213 \\
\hline \multicolumn{13}{|l|}{ SUV $_{\max }$ sum } \\
\hline All LNs at diagnosis & 17.2 & $10.4-30.6$ & 0.98 & 0.365 & 0.99 & 0.903 & 0.98 & 0.632 & 1.01 & 0.468 & 0.98 & 0.374 \\
\hline All LNs after RT & 6.3 & $3.3-9.5$ & 1.01 & 0.776 & 1.09 & 0.065 & 1.05 & 0.374 & 1.29 & 0.007 & 0.99 & 0.950 \\
\hline Reduction (\%) in all LNs & 60 & $43-80$ & 0.20 & 0.098 & 0.45 & 0.321 & 0.41 & 0.408 & 0.55 & 0.332 & 0.32 & 0.337 \\
\hline \multicolumn{13}{|l|}{ Target lesion $^{\dagger}$ SUV $_{\max }$} \\
\hline At diagnosis & 15.4 & $11.1-17.9$ & 1.04 & 0.264 & 1.01 & 0.802 & 1.00 & 0.982 & 0.99 & 0.938 & 0.97 & 0.645 \\
\hline After RT & 3.8 & $2.7-5.2$ & 1.27 & $<0.0001$ & 1.17 & 0.003 & 1.19 & 0.004 & 1.17 & 0.004 & 1.17 & 0.044 \\
\hline Reduction (\%) & 72 & $55-80$ & 0.06 & 0.002 & 0.03 & 0.001 & 0.06 & 0.007 & 0.04 & 0.002 & 0.07 & 0.028 \\
\hline \multicolumn{13}{|c|}{$\begin{array}{l}\text { Sum of primary tumor }+ \text { LNs } \\
\text { SUV }_{\max }\end{array}$} \\
\hline At diagnosis & 24.4 & $15.1-38.3$ & 0.98 & 0.422 & 1.00 & 0.678 & 0.99 & 0.524 & 1.01 & 0.622 & 0.99 & 0.745 \\
\hline After RT & 7.9 & $5.9-13.9$ & 1.06 & 0.083 & 1.13 & 0.001 & 1.06 & 0.091 & 1.13 & 0.001 & 1.08 & 0.115 \\
\hline Reduction (\%) & 65 & $45-74$ & 0.04 & 0.004 & 0.06 & 0.033 & 0.06 & 0.020 & 0.07 & 0.021 & 0.11 & 0.163 \\
\hline
\end{tabular}

${ }^{*}$ Regional LN with the highest SUV $\max$.

${ }^{\dagger}$ Target lesion (either primary tumor or LN) with the highest SUV $V_{\max }$.

Values shown are hazard ratios.

reduction between the primary tumor (median, 70\%) and the regional LN with the highest $\mathrm{SUV}_{\text {max }}$ after RT (median, $64 \% ; P=0.384)$. We found that, after RT, the higher the uptake was in the single LN with the highest post-RT $\mathrm{SUV}_{\text {max }}$, the shorter were the OS time $(P=0.004$; Fig. 2$)$, the LRC inside the radiation field $(P=0.001)$, the LRC both inside and outside the radiation field $(P=0.002)$, and the DFS time $(P=0.001$; Fig. 4). Figures 4 and 5 illustrate the effect of $\mathrm{SUV}_{\max }$ on OS and DFS, respectively, with the cut-off point of the $\mathrm{SUV}_{\max }$ after RT set at 3.1 (the 50th percentile for LNs); patients with an $\mathrm{SUV}_{\max }$ of less than 3.1 also had better OS and DFS than did those with an $\mathrm{SUV}_{\max }$ of 3.1 or more (OS, $P=0.0143$; DFS, $P=0.0028$ ). For the summed $\mathrm{SUV}_{\max }$ of all PETpositive LNs, only the uptake after RT was significantly associated with LRC inside and outside the radiation field $(P=0.007)$. The results also indicated that the higher the summed $\mathrm{SUV}_{\max }$ was after RT, the shorter was the LRC. These associations all retained significance in multivariate analysis (Table 3). Again, the $\mathrm{SUV}_{\max }$ at diagnosis did not correlate with any of the measured outcomes.

$S U V_{\text {max }}$ for Target Lesion (Either Primary Tumor or Regional LNs). The target lesion with the highest $\mathrm{SUV}_{\max }$ at diagnosis was the primary tumor in $67 \%$ of cases and the LNs in $33 \%$. Similar results were observed after RT, when the highest $\mathrm{SUV}_{\max }$ was the primary tumor in $63 \%$ of cases and the LNs in $37 \%$ of cases. The higher the post-RT $\mathrm{SUV}_{\text {max }}$ of the single target lesion with the highest post-

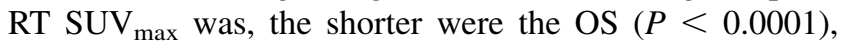
DFS $(P=0.003)$, LRC inside the radiation field $(P=$ $0.004)$, LRC both inside and outside the radiation field $(P=0.004)$, and DMFS $(P=0.044$; Table 2$)$. Findings were similar for the extent of $\mathrm{SUV}_{\max }$ reduction after RT; specifically, the greater the $\mathrm{SUV}_{\text {max }}$ reduction was after RT in the single target lesion with the highest $\mathrm{SUV}_{\max }$ at diagnosis, the longer were the OS ( $P=0.002$; Fig. 2$)$, DFS $(P=0.001$; Fig. 3$)$, LRC inside the radiation field $(P=$ $0.007)$, LRC both inside and outside the radiation field $(P=$ $0.002)$, and DMFS ( $P=0.028$; Table 2$)$. Figures 4 and 5 illustrate the effect of the post-RT $\mathrm{SUV}_{\max }$ of the single target lesion with the highest post-RT SUV max $_{\text {and }}$ and the extent of $\mathrm{SUV}_{\max }$ reduction after RT on OS and DFS when dichotomized according to the 50th percentile of the corresponding variable. Patients with a post-RT SUV max $_{\text {of less }}$ than 3.8 or an $\mathrm{SUV}_{\max }$ reduction of $72 \%$ or more had better OS and DFS than did those with a post-RT SUV max $_{\text {of }} 3.1$ or more (OS, $P=0.0006$; DFS, $P=0.0045$ ) or an $\mathrm{SUV}_{\max }$ reduction of less than $72 \%$ (OS, $P=0.0074$; DFS, $P=$ 0.0603). However, the pretreatment $\mathrm{SUV}_{\max }$ was not associated with any survival outcome. When analyzing the summed $\mathrm{SUV}_{\max }$ of the primary tumor plus all PET-positive LNs, we observed that the higher the values were after 


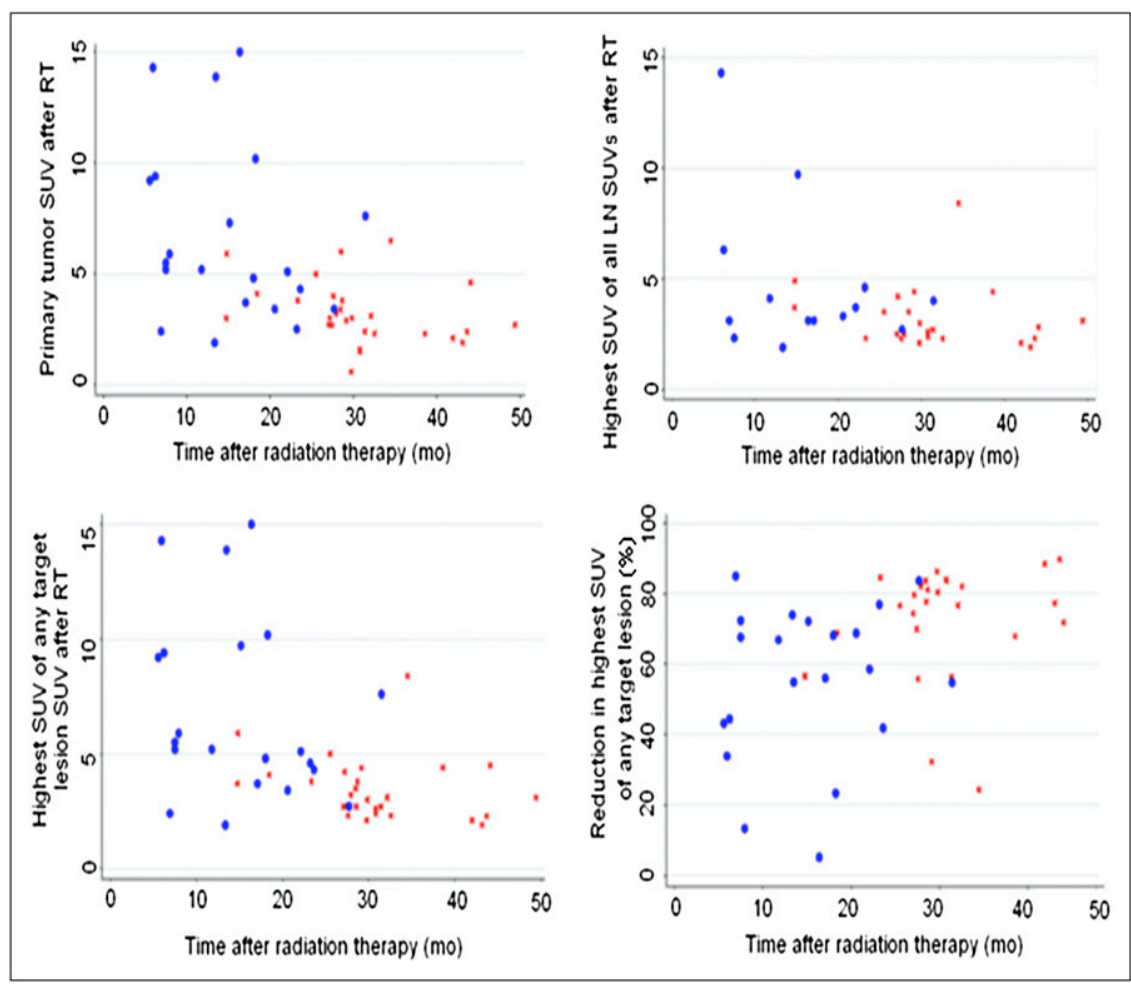

FIGURE 2. Scatterplot of OS time ( $x$-axis) vs. SUV $V_{\max }$ after radiotherapy for patients alive (red symbols) and or dead (blue symbols).
RT, the shorter were the LRC both inside and outside the radiation field $(P=0.001)$ and the DFS $(P=0.001)$. Finally, we found that the greater the reduction was in the summed $\mathrm{SUV}_{\max }$ of the primary tumor plus all PET-positive LNs, the longer were the OS $(P=0.004)$, LRC inside and outside the field $(P=0.021)$, and DFS $(P=0.033$; Table 2). All of these results, except for the association between the reduction in the summed $\mathrm{SUV}_{\max }$ of the primary tumor plus all PET-positive LNs and OS, retained significance in multivariate analyses (Table 3 ).

\section{DISCUSSION}

PET/CT images provide functional information about cancer by assessing the uptake of a radioactive glucose

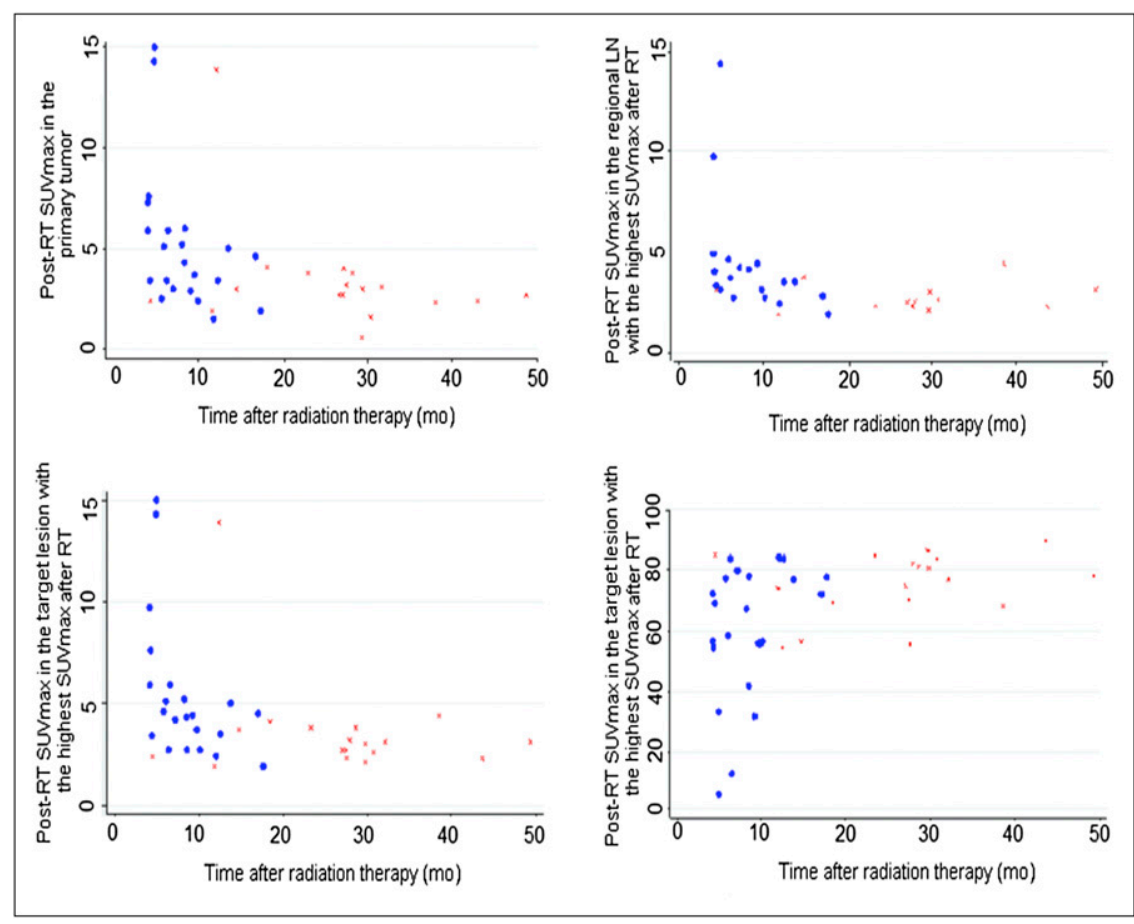

FIGURE 3. Scatterplot of DFS time ( $x$-axis) vs. SUV $V_{\max }$ after radiotherapy for patients with (red symbols) and without (blue symbols) recurrent disease. 
FIGURE 4. OS according to 50th percentile of SUV $_{\max }$ after radiotherapy. "Total number of patients differs from other figures because 4 patients were stage N0 disease, and in $8 \mathrm{~N}$-positive stage cases, it was difficult to distinguish each primary tumor accumulation of ${ }^{18} \mathrm{~F}-\mathrm{FDG}$ from its $\mathrm{LN}$ accumulation. Thus, ${ }^{18} \mathrm{~F}-\mathrm{FDG}$ accumulation was regarded as $\mathrm{SUV}_{\max }$ for primary tumor.

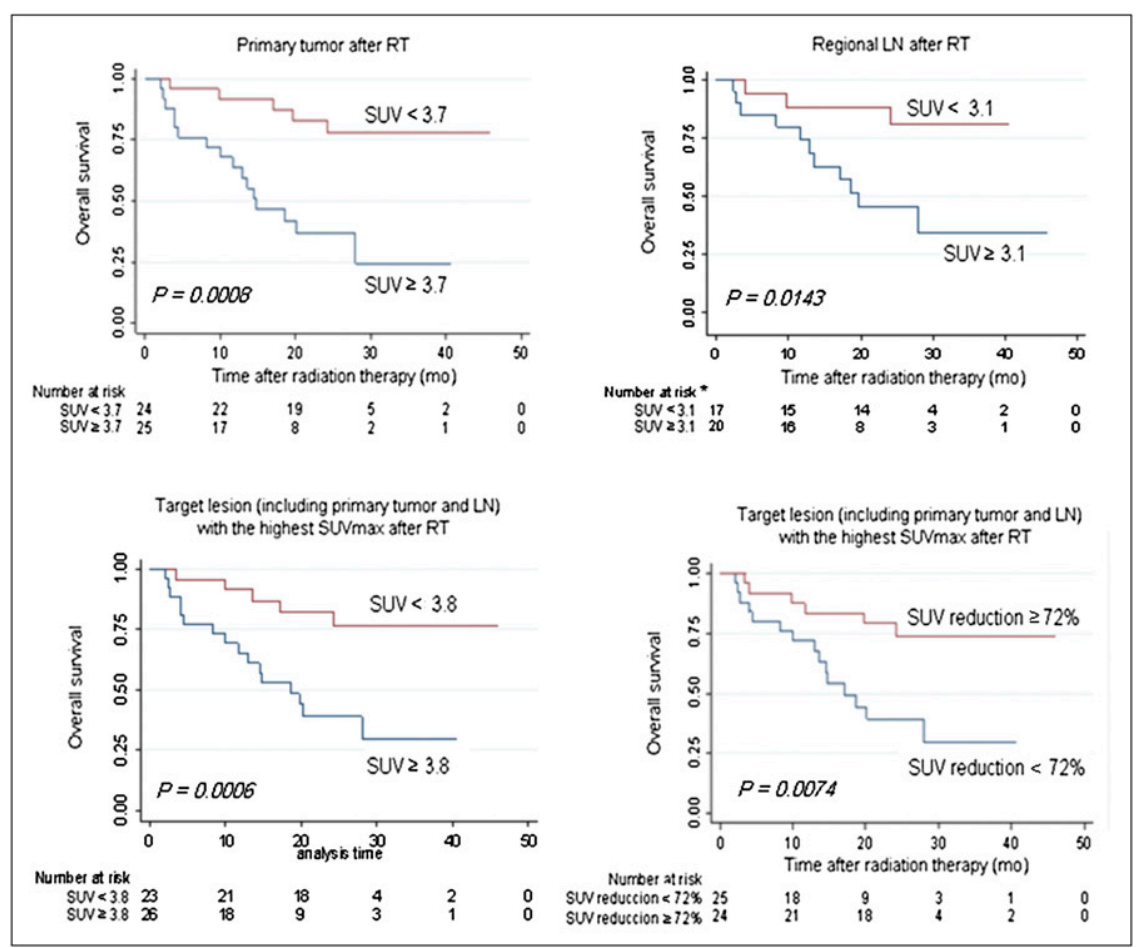

analog by a tumor-a process that most likely reflects the biologic behavior of a particular case of cancer in terms of proliferation, metastatic potential, sensitivity to therapy, and more $(18,19)$. The key findings of the current study were that the regional LN with the highest $\mathrm{SUV}_{\max }$ after RT was sig- nificantly associated with OS: the lower the post-RT SUV $\mathrm{max}_{\max }$ was, the longer the OS was. Our results also showed that the extent of reduction in $\mathrm{SUV}_{\max }$ was associated with disease control after curative RT for locally advanced NSCLC: the greater the decrease in $\mathrm{SUV}_{\max }$ was in the
FIGURE 5. DFS according to 50th percentile of $S V_{\text {max }}$ after radiotherapy. "Total number of patients differs from other figures because 4 patients were stage N0 disease, and in $8 \mathrm{~N}$-positive stage cases, it was difficult to distinguish each primary tumor accumulation of ${ }^{18} \mathrm{~F}-\mathrm{FDG}$ from its $\mathrm{LN}$ accumulation. Thus, ${ }^{18} \mathrm{~F}-\mathrm{FDG}$ accumulation was regarded as SUV $\mathrm{V}_{\max }$ for primary tumor.

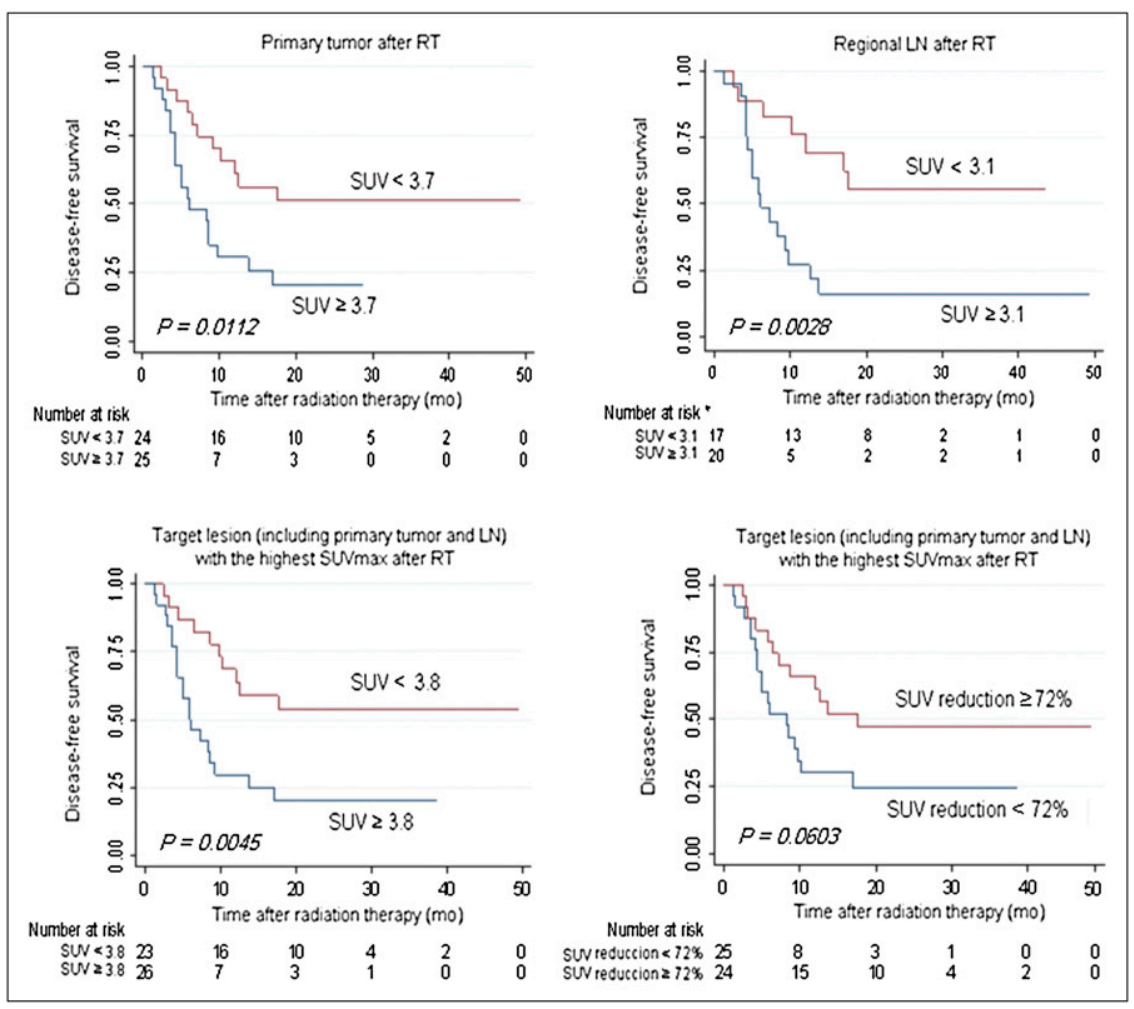


TABLE 3

Multivariate Analyses of SUVs and Survival

\begin{tabular}{|c|c|c|c|c|c|c|c|c|c|c|}
\hline \multirow[b]{2}{*}{ Variable } & \multicolumn{2}{|c|}{ OS } & \multicolumn{2}{|c|}{ DFS } & \multicolumn{2}{|c|}{ LRC inside field } & \multicolumn{2}{|c|}{$\begin{array}{l}\text { LRC inside }+ \\
\text { outside field }\end{array}$} & \multicolumn{2}{|c|}{$\begin{array}{l}\text { Distant } \\
\text { metastasis- } \\
\text { free survival }\end{array}$} \\
\hline & $\begin{array}{l}\text { Hazard } \\
\text { ratio }\end{array}$ & $P$ & $\begin{array}{l}\text { Hazard } \\
\text { ratio }\end{array}$ & $P$ & $\begin{array}{l}\text { Hazard } \\
\text { ratio }\end{array}$ & $P$ & $\begin{array}{l}\text { Hazard } \\
\text { ratio }\end{array}$ & $P$ & $\begin{array}{l}\text { Hazard } \\
\text { ratio }\end{array}$ & $P$ \\
\hline Primary tumor SUV max $_{\text {after RT }}$ & 1.31 & $<0.0001$ & 1.30 & $<0.0001$ & 1.19 & $0.002^{*}$ & 1.35 & $<0.0001$ & & - \\
\hline Regional $\mathrm{LN}^{\dagger} \mathrm{SUV}_{\max }$ after RT & 1.63 & $<0.0001$ & 1.32 & $0.001^{\star}$ & 1.58 & 0.003 & 1.55 & $<0.0001$ & & - \\
\hline SUV $_{\max }$ sum of all LNs after RT & & - & & - & & - & 1.43 & $<0.0001$ & & - \\
\hline Target lesion ${ }^{\ddagger}$ SUV $_{\max }$ after RT & 1.40 & $<0.0001$ & 1.33 & $<0.0001$ & 1.19 & $0.004^{*}$ & 1.38 & $<0.0001$ & 1.17 & $0.044^{*}$ \\
\hline Reduction (\%) in target lesion ${ }^{\ddagger}$ SUV $_{\max }$ & 0.07 & 0.013 & 0.03 & $0.001^{*}$ & 0.06 & $0.007^{*}$ & NA & & 0.07 & $0.028^{*}$ \\
\hline $\begin{array}{l}\text { Sum of primary tumor }+ \text { LNs } \text { SUV }_{\max } \\
\text { after RT }\end{array}$ & & - & 1.15 & $<0.0001$ & & - & 1.19 & $<0.0001$ & & - \\
\hline $\begin{array}{l}\text { Reduction (\%) in sum of primary } \\
\text { tumor }+\mathrm{LNs}_{\mathrm{SUV}} \mathrm{V}_{\max }\end{array}$ & 0.10 & 0.053 & 0.06 & $0.033^{\star}$ & 0.06 & $0.020^{\star}$ & NA & & & - \\
\hline
\end{tabular}

Variables shown are those that had statistical significance on univariate analysis.

*After stepwise backward elimination procedure, only this variable showed significance. Therefore, no other covariables could be included in final model for adjustment.

${ }^{\dagger}$ Regional LN with highest SUV max $_{\text {. }}$

‡Target lesion (either primary tumor or LN) with highest SUV $\mathrm{Fax}_{\text {. }}$

lesion (primary tumor or $\mathrm{LN}$ ) that had the highest $\mathrm{SUV}_{\max }$ at diagnosis, the longer was the OS. We further found that the lower the post-RT SUV ${ }_{\max }$ was in the primary tumor or the regional LN with the highest $\mathrm{SUV}_{\text {max }}$ after RT, the longer were the DFS and LRC times. Finally, the greater the decline in $\mathrm{SUV}_{\text {max }}$ was after RT of the lesion with the highest $\mathrm{SUV}_{\max }$ at diagnosis, the longer was the DMFS time.

It is well established that patients with inoperable NSCLC showing residual metabolic activity within the primary tumor after radiochemotherapy have worse OS than patients without such residual activity (18). Our results are consistent with data published by others. Several investigators have explored the usefulness of pretreatment ${ }^{18} \mathrm{~F}-\mathrm{FDG}$ PET for predicting outcome for patients with NSCLC, and several cut-off SUV levels have been proposed $(13,19,20)$. However, other recent studies $(14,21)$ have shown that pretreatment ${ }^{18} \mathrm{~F}-\mathrm{FDG}$ uptake of the primary tumor in patients with newly diagnosed advanced-stage NSCLC was not related to survival. For instance, Ikushima et al. (21) evaluated the relationship between the $\mathrm{SUV}_{\max }$ and clinical tumor features in 149 patients with NSCLC who underwent pretreatment PET $(n=67)$ or PET/CT $(n=82)$ and definitive RT. A high $\mathrm{SUV}_{\text {max }}$ was a negative factor for LRC, DMFS, and OS on univariate analysis in the PET group, but the significance decreased when tumor size was included in the analysis. In addition, a high $\mathrm{SUV}_{\max }$ was not a negative factor for LRC, DMFS, or OS in the PET/CT group, suggesting that the evidence was not sufficient to conclude that pretreatment ${ }^{18}$ F-FDG uptake in a primary NSCLC tumor provides prognostic information. Consistent with these findings, we did not observe a significant relationship between $\mathrm{SUV}_{\max }$ before RT and survival.

Therefore, the post-RT SUV $\mathrm{Sax}_{\max }$ or the extent of reduction in ${ }^{18}$ F-FDG PET SUV max $_{\text {ax }}$ after treatment may be a better method of predicting survival than the pretreatment values. This supposition is supported by several recent reports $(22,23)$. For instance, Aerts et al. (22) found that patients with residual metabolically active areas within the primary tumor after RT had significantly worse survival than did individuals whose tumors showed a complete metabolic response. In our study, we found that higher post-RT SUV $\mathrm{max}_{\max }$ not only in the primary tumor but also in the regional LN with the highest residual $\mathrm{SUV}_{\max }$ after RT were strong negative prognostic factors for survival. This finding in the regional LN was a novel discovery. In addition, the association found between the post-RT SUV $\mathrm{max}_{\text {max }}$ and OS noted for patients who had PET scans more than 2.5 mo after the end of RT (but not for those who had earlier PET scans) suggests that scans done sooner than 2.5 mo after RT may not reflect much of the effect of the RT.

The utility of the SUV for disease staging or its role after neoadjuvant chemotherapy for NSCLC has been described before $(3,24-27)$. However, the usefulness of the extent of reduction in SUV after definitive RT, particularly in LNs, for assessing prognosis remains uncertain. In our analysis, neither the reduction in $\mathrm{SUV}_{\max }$ in the primary tumor nor the reduction in the LNs was associated with any survival outcome assessed. However, when evaluating the percentage of $\mathrm{SUV}_{\max }$ reduction after RT in the target lesion (either primary tumor or $\mathrm{LN}$ ) with the highest $\mathrm{SUV}_{\max }$ at diagnosis, we found a significant association with OS. This result highlights the role of the most ${ }^{18} \mathrm{~F}-\mathrm{FDG}$-avid lesion, regardless of whether that lesion is the primary tumor or the LNs, in predicting outcome after treatment of locally advanced NSCLC.

Greene et al. (16) proposed that a complete response after high-dose RT or concurrent chemoradiotherapy is defined as the complete disappearance of all evidence of ma- 
lignant disease or residual radiographic abnormalities at 3 and 6 mo after completion of RT that then remains stable for an additional 6 mo or more. A recent Dutch study (18) confirmed the validity of metabolic response assessment up to 6 mo after RT as a surrogate of survival. To predict survival and obtain this information to help identify patients who are at high risk of recurrence and to design additional salvage treatment early, we decided to evaluate the first PET scan done after RT, which usually took place 1-3 mo after treatment, and found that we could indeed obtain this information as early as 3 mo after RT. Whether patients with residual ${ }^{18} \mathrm{~F}-\mathrm{FDG}$ uptake after RT should be offered additional treatment is still uncertain, and this question should be prospectively evaluated in protocols to evaluate further management strategies such as new targeted therapies or adjuvant chemotherapy for such patients. Currently, the American College of Radiology Imaging Network 6668/RTOG 0235 trial is prospectively evaluating whether the primary tumor ${ }^{18} \mathrm{~F}$-FDG $\mathrm{SUV}_{\max }$ shortly after definitive chemoradiation can predict long-term survival in inoperable stage II or III NSCLC. This multiinstitutional study will clarify whether our findings are also observed in a larger sample.

On the basis of our findings and others $(13,18,23)$, we could speculate that tumor glucose metabolism is related to the metastatic potential of the tumor. Increased ${ }^{18} \mathrm{~F}-\mathrm{FDG}$ uptake on PET images may correlate with conventional tissue markers of tumor aggressiveness, markers of hypoxia or angiogenesis, or molecular markers of proliferation, such as $\mathrm{Ki}-67(28,29)$. The overexpression of various molecular markers of tumor biology in common solid tumors such as NSCLC may be associated with PET findings, clinical outcome, or both, which would provide a new avenue for targeted therapies (30).

\section{CONCLUSION}

We found that the post-RT SUV $\mathrm{max}_{\max }$ for the primary tumor and LNs, as well as the extent of reduction after RT in the target lesion that had the highest $\mathrm{SUV}_{\max }$ at diagnosis, were associated with OS and DFS in patients with NSCLC. These findings indicate that the post-RT SUV $\mathrm{Sax}_{\max }$ and the extent of reduction may become a routinely used, quantifiable, and analytic indicator of disease activity and treatment efficacy.

\section{DISCLOSURE STATEMENT}

The costs of publication of this article were defrayed in part by the payment of page charges. Therefore, and solely to indicate this fact, this article is hereby marked "advertisement" in accordance with 18 USC section 1734.

\section{ACKNOWLEDGMENTS}

We thank Tinsu Pan, Lawrence Levy, and Christine Wogan for their contributions to this report. Financial support for this research was provided by M.D. Anderson
Cancer Center. No other potential conflict of interest relevant to this article was reported.

\section{REFERENCES}

1. O'Rourke N, Macbeth F. Is concurrent chemoradiation the standard of care for locally advanced non-small cell lung cancer? A review of guidelines and evidence. Clin Oncol (R Coll Radiol). 2010;22:347-355.

2. Erasmus JJ, Rohren E, Swisher SG. Prognosis and reevaluation of lung cancer by positron emission tomography imaging. Proc Am Thorac Soc. 2009;6:171-179.

3. Lee HY, Lee HJ, Kim YT, et al. Value of combined interpretation of computed tomography response and positron emission tomography response for prediction of prognosis after neoadjuvant chemotherapy in non-small cell lung cancer. $J$ Thorac Oncol. 2010;5:497-503.

4. Malone JP, Gerberi MA, Vasireddy S, et al. Early prediction of response to chemoradiotherapy for head and neck cancer: reliability of restaging with combined positron emission tomography and computed tomography. Arch Otolaryngol Head Neck Surg. 2009;135:1119-1125.

5. Meignan M, Itti E, Gallamini A, Haioun C. Interim ${ }^{18} \mathrm{~F}$-fluorodeoxyglucose positron emission tomography in diffuse large B-cell lymphoma: qualitative or quantitative interpretation-where do we stand? Leuk Lymphoma. 2009;50: 1753-1756.

6. Schreyögg J, Weller J, Stargardt T, et al. Cost-effectiveness of hybrid PET/CT for staging of non-small cell lung cancer. J Nucl Med. 2010;51:1668-1675.

7. Specht JM, Kurland BF, Montgomery SK, et al. Tumor metabolism and blood flow as assessed by positron emission tomography varies by tumor subtype in locally advanced breast cancer. Clin Cancer Res. 2010;16:2803-2810.

8. Hellwig D, Graeter TP, Ukena D, et al. ${ }^{18} \mathrm{~F}-\mathrm{FDG}$ PET for mediastinal staging of lung cancer: which SUV threshold makes sense? J Nucl Med. 2007;48:17611766 .

9. Feng M, Kong FM, Gross M, Fernando S, Hayman JA, Ten Haken RK. Using fluorodeoxyglucose positron emission tomography to assess tumor volume during radiotherapy for non-small-cell lung cancer and its potential impact on adaptive dose escalation and normal tissue sparing. Int J Radiat Oncol Biol Phys. 2009; 73:1228-1234.

10. Wu K, Ung YC, Hwang D, et al. Autocontouring and manual contouring: which is the better method for target delineation using ${ }^{18} \mathrm{~F}-\mathrm{FDG}$ PET/CT in non-small cell lung cancer? J Nucl Med. 2010;51:1517-1523.

11. Keidar Z, Haim N, Guralnik L, et al. PET/CT using ${ }^{18}$ F-FDG in suspected lung cancer recurrence: diagnostic value and impact on patient management. $\mathrm{J} \mathrm{Nucl}$ Med. 2004;45:1640-1646.

12. Downey RJ, Akhurst T, Gonen M, Park B, Rusch V. Fluorine-18 fluorodeoxyglucose positron emission tomographic maximal standardized uptake value predicts survival independent of clinical but not pathologic TNM staging of resected non-small cell lung cancer. J Thorac Cardiovasc Surg. 2007;133:1419-1427.

13. Sasaki R, Komaki R, Macapinlac H, et al. $\left[{ }^{18} \mathrm{~F}\right]$ fluorodeoxyglucose uptake by positron emission tomography predicts outcome of non-small-cell lung cancer. J Clin Oncol. 2005;23:1136-1143.

14. Hoang JK, Hoagland LF, Coleman RE, Coan AD, Herndon JE, 2nd, Patz EF Jr. Prognostic value of fluorine-18 fluorodeoxyglucose positron emission tomography imaging in patients with advanced-stage non-small-cell lung carcinoma. J Clin Oncol. 2008;26:1459-1464.

15. Hicks RJ. Role of ${ }^{18}$ F-FDG PET in assessment of response in non-small cell lung cancer. J Nucl Med. 2009;50(suppl 1):31S-42S.

16. Greene FL, Page DL, Fleming ID, et al., eds. Lung. In: AJCC Cancer Staging Manual, 6th ed. New York, NY: Springer; 2002.

17. Soret M, Bacharach SL, Buvat I. Partial-volume effect in PET tumor imaging. J Nucl Med. 2007;48:932-945.

18. Velazquez ER, Aerts HJ, Oberije C, De Ruysscher D, Lambin P. Prediction of residual metabolic activity after treatment in NSCLC patients. Acta Oncol. 2010;49:1033-1039.

19. Goodgame B, Pillot GA, Yang Z, et al. Prognostic value of preoperative positron emission tomography in resected stage I non-small cell lung cancer. $J$ Thorac Oncol. 2008;3:130-134.

20. Hanin FX, Lonneux M, Cornet J, et al. Prognostic value of FDG uptake in early stage non-small cell lung cancer. Eur J Cardiothorac Surg. 2008;33:819-823.

21. Ikushima H, Dong L, Erasmus J, et al. Predictive value of ${ }^{18} \mathrm{~F}$-fluorodeoxyglucose uptake by positron emission tomography for non-small cell lung cancer patients treated with radical radiotherapy. J Radiat Res (Tokyo). 2010;51:465471.

22. Aerts HJ, van Baardwijk AA, Petit SF, et al. Identification of residual metabolicactive areas within individual NSCLC tumours using a pre-radiotherapy ${ }^{18}$ fluorodeoxyglucose-PET-CT scan. Radiother Oncol. 2009;91:386-392. 
23. van Loon J, Grutters J, Wanders R, et al. Follow-up with 18FDG-PET-CT after radical radiotherapy with or without chemotherapy allows the detection of potentially curable progressive disease in non-small cell lung cancer patients: a prospective study. Eur J Cancer. 2009;45:588-595.

24. Billé A, Pelosi E, Skanjeti A, et al. Preoperative intrathoracic lymph node staging in patients with non-small-cell lung cancer: accuracy of integrated positron emission tomography and computed tomography. Eur J Cardiothorac Surg. 2009;36:440-445.

25. Liu BJ, Dong JC, Xu CQ, et al. Accuracy of ${ }^{18}$ F-FDG PET/CT for lymph node staging in non-small-cell lung cancers. Chin Med J (Engl). 2009;122:1749-1754.

26. Aukema TS, Kappers I, Olmos RA, et al. Is ${ }^{18}$ F-FDG PET/CT useful for the early prediction of histopathologic response to neoadjuvant erlotinib in patients with non-small cell lung cancer? J Nucl Med. 2010;51:1344-1348.
27. de Geus-Oei LF, van der Heijden HF, Visser EP, et al. Chemotherapy response evaluation with ${ }^{18}$ F-FDG PET in patients with non-small cell lung cancer. $\mathrm{J} \mathrm{Nucl}$ Med. 2007;48:1592-1598.

28. Bos R, van Der Hoeven JJ, van Der Wall E, et al. Biologic correlates of ${ }^{18}$ fluorodeoxyglucose uptake in human breast cancer measured by positron emission tomography. J Clin Oncol. 2002;20:379-387.

29. Vesselle H, Schmidt RA, Pugsley JM, et al. Lung cancer proliferation correlates with [F-18]fluorodeoxyglucose uptake by positron emission tomography. Clin Cancer Res. 2000;6:3837-3844.

30. Jadvar H, Alavi A, Gambhir SS. ${ }^{18} \mathrm{~F}-\mathrm{FDG}$ uptake in lung, breast, and colon cancers: molecular biology correlates and disease characterization. $\mathrm{J} \mathrm{Nucl}$ Med. 2009;50:1820-1827. 